\title{
EchoGéo
}

\section{La carte municipale post-apartheid : justice sociospatiale et innovations territoriales post- modernes}

Frédéric Giraut

\section{CpenEdition}

Journals

Édition électronique

URL : https://journals.openedition.org/echogeo/12098

DOI : 10.4000/echogeo.12098

ISSN : 1963-1197

Éditeur

Pôle de recherche pour l'organisation et la diffusion de l'information géographique (CNRS UMR 8586)

Référence électronique

Frédéric Giraut, " La carte municipale post-apartheid : justice sociospatiale et innovations territoriales post-modernes », EchoGéo [En ligne], 13 | 2010, mis en ligne le 14 septembre 2010, consulté le 03 août 2021. URL : http://journals.openedition.org/echogeo/12098; DOI : https://doi.org/10.4000/echogeo. 12098

Ce document a été généré automatiquement le 3 août 2021.

EchoGéo est mis à disposition selon les termes de la licence Creative Commons Attribution - Pas d'Utilisation Commerciale - Pas de Modification 4.0 International (CC BY-NC-ND) 


\section{La carte municipale post-apartheid: justice sociospatiale et innovations territoriales post-modernes}

\section{Frédéric Giraut}

Illustration 1 - Entrée du Settlement de Botshabelo, municipalité de Mangung

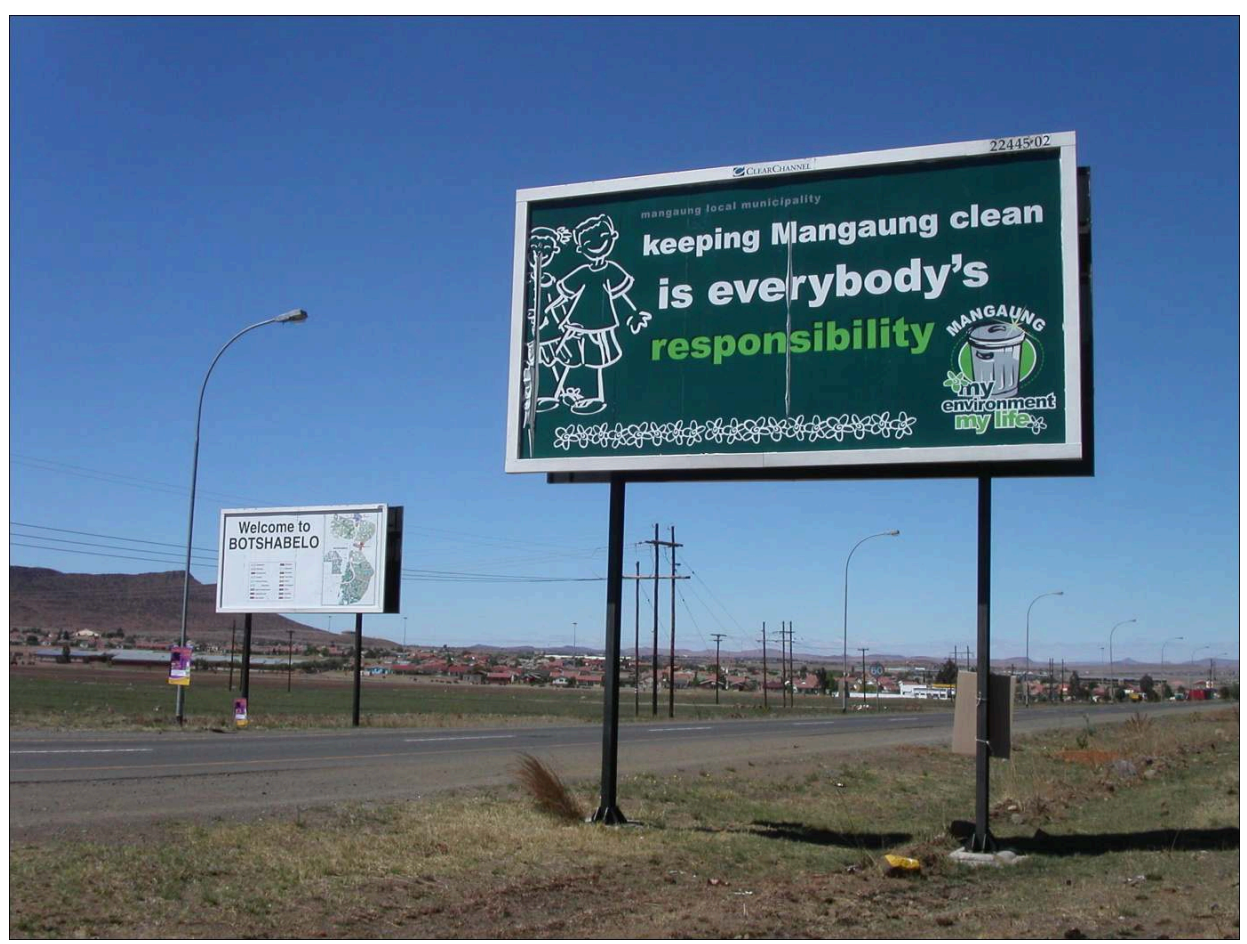

Auteur : Frédéric Giraut, 2003.

1 Après avoir éliminé les bantoustans et refondu le découpage provincial, les dirigeants de la Nouvelle Afrique du Sud se sont appliqués à mettre en place un système municipal égalitaire et rationnel. Un organisme placé directement sous l'autorité de la Présidence, 
le Municipal Demarcation Board, dirigé par un géographe, Dr. Michael Sutcliffe, fut chargé de mener à bien le processus. Le principe de mixité socio-spatiale associé à celui de pavage intégral (Wall to wall) et d'emboîtement (province, municipalité de district et municipalité locale) furent les maîtres mots du projet. Cependant, des exceptions notables ont confirmé la règle et dérogent aux principes de cette rationalité. Elles concernent d'une part les aires métropolitaines qui s'autonomisent au sein du système municipal et provincial, et d'autre part les espaces aux marges du nouveau découpage provincial pour lesquels ont été créées les formules hybrides du District Management Area (DMA) et de la Cross Boundaries Municipality (CBLC et CBDC) (illustrations 2 et 3).

Illustration 2 - Carte des nouvelles municipalités sud-africaines par province

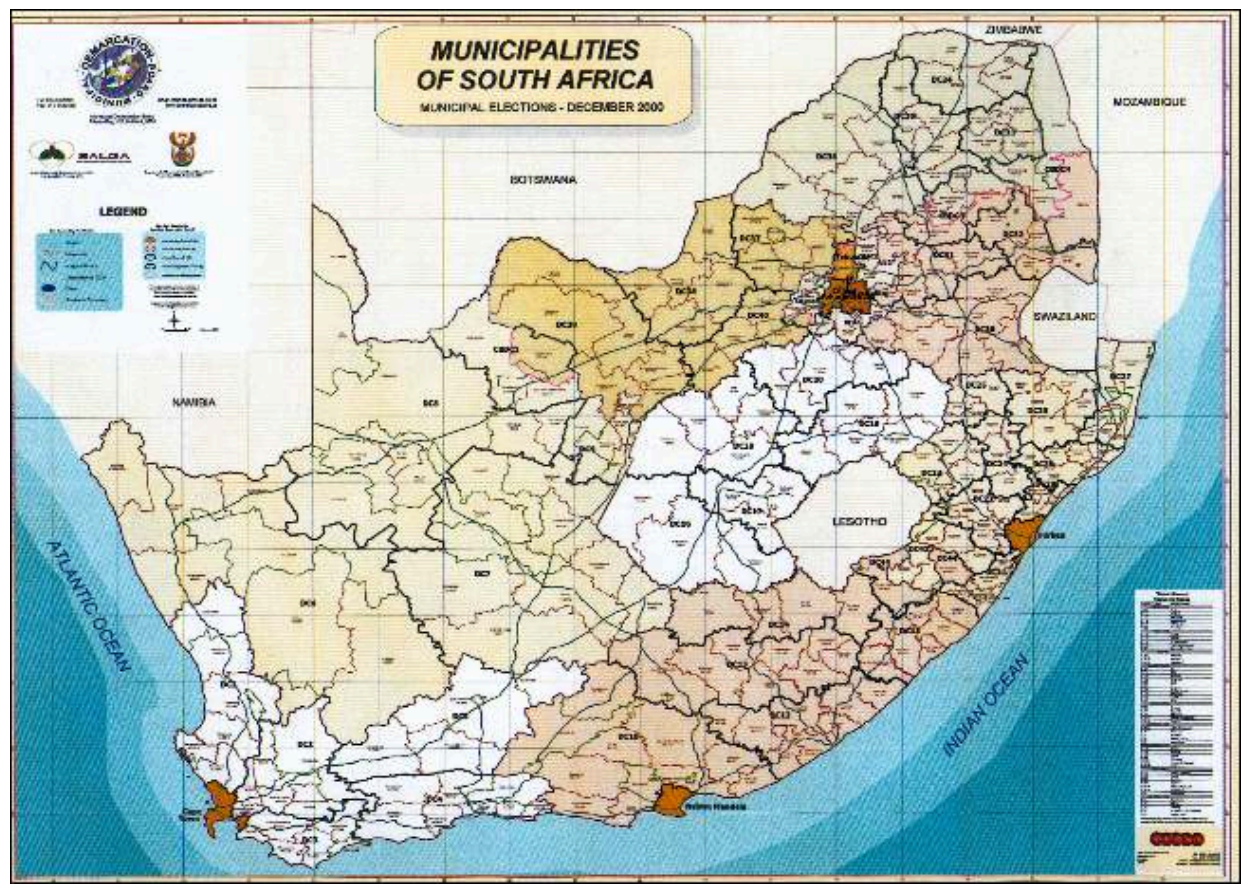

Source : Municipal Demarcation Board, 2000 
Illustration 3 - Les statuts municipaux spécifiques

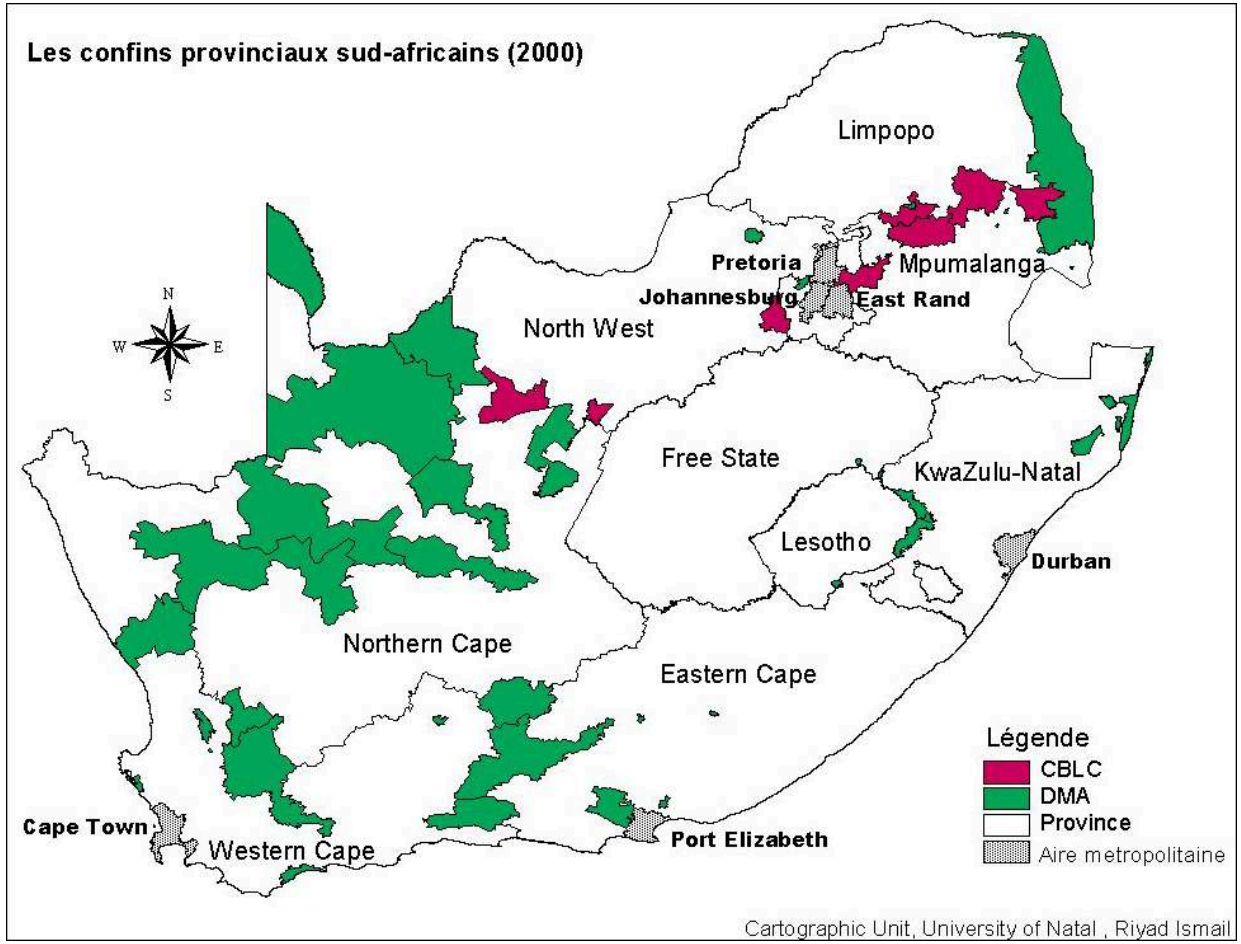

Auteurs: R. Ismail, F. Giraut, B. Antheaume, Cartographic Unit University of Natal, 2001.

2 Le régime général est donc celui d'un double niveau de municipalités composites. Chaque municipalité associe autant que faire se peut quatre types d'espaces très contrastés issus des divisions socio-spatiales de la colonisation puis de l'apartheid (illustration 4):

- les parties privilégiées des agglomérations, c'est à dire les CBD et les suburbs blanches du temps de l'apartheid et qui seuls constituaient les municipalités ;

- les townships séparés des précédents par des zones tampons (buffer zones);

- les campagnes denses des anciens bantoustans incluant les concentrations humaines, véritables fragments urbains projetés au delà des frontières de ces anciens pseudo-États ; - les espaces agricoles et forestiers d'origine coloniale. 
Illustration 4 - Les formes d'urbanisation et de peuplement en Afrique du Sud au sortir de l'apartheid (projection en 2000)

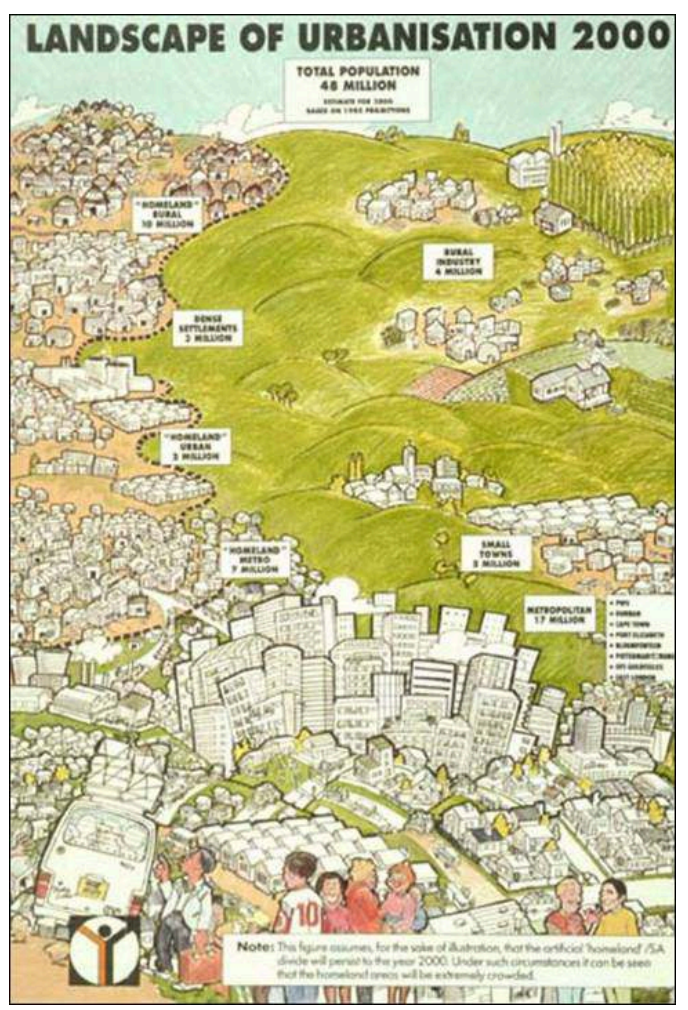

Source : The Urban Foundation, 1990.

Le défi est important et les résistances furent nombreuses, du côté des autorités coutumières (Tribal Authorities) notamment dans le KwaZulu-Natal (illustration 5) ou encore de la part de notables locaux à la légitimité politique issue de la lutte contre l'apartheid et qui ne voulaient pas voir leur territoire marginal se fondre dans de vastes entités composites dominées par les villes et leurs townships. Ces réticences ont été surmontées pour la plupart et de vastes municipalités ont vu le jour qui incluent des fragments urbains situés à plusieurs dizaines de kilomètres. C'est le cas ici (illustration 1) avec la nouvelle municipalité de Mangaung (Bloemfontein), du nom du principal township de Bloemfontein, qui s'étend jusqu'à Botshabelo fragment urbain projeté à près de cinquante kilomètres de la ville principale. Ce settlement était destiné dans le cadre du Grand apartheid à rejoindre un bantoustan, en l'occurrence le QwaQwa des Sotho puisque les résidents originels de Botshabelo étaient avant tout des expulsés sotho des townships de Bloemfontein et des expulsés sotho du fragment voisin du bantoustan du Bophuthatswana en phase d'épuration ethnique. 
Illustration 5 - Le Municipal Demarcation Board aux prises avec les Tribal authorities zouloues dans le KwaZulu-Natal

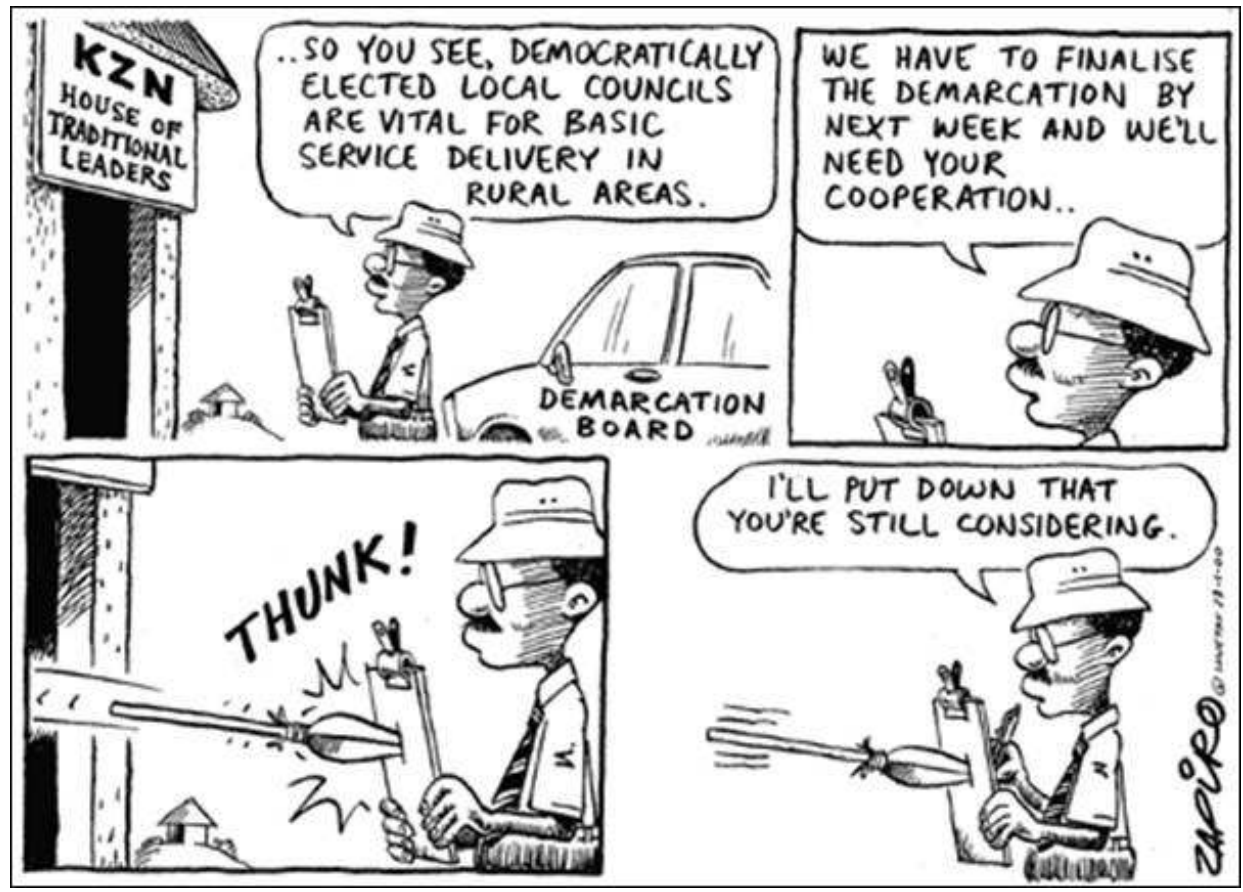

Source : dessin de Zapiro paru le 27 janvier 2000 dans le quotidien national The Sowetan

4 L'opération post apartheid, souvent réussie, de constitution de municipalités composites s'apparente à une rationalisation de l'espace administratif et à un traitement territorial de type moderne en lieu et place d'un système inégalitaire qui jouait sur la diversité des statuts en lien avec la hiérarchie des lieux et des communautés.

Les immenses attentes que le renouveau municipal a suscitées en termes de services de base pour les populations et les quartiers démunis (illustration 6) ont cependant entraîné des dérogations à la norme et la mise en place sur certaines portions du territoire de grandes municipalités uniques qui cumulent les fonctions des deux niveaux normalement emboîtés. C'est le cas des aires de très faibles densités des régions arides et des vastes aires protégées dotées d'un seul statut de district management area, où la notion de municipalité de base fondée sur la proximité relative n'a pas de sens et où il s'agit de gérer de l'étendue plus que des communautés nombreuses et composites. C'est le cas aussi et surtout des principales agglomérations du pays dotées d'un statut unique de super municipalités métropolitaines (six au total dont trois pour la vaste conurbation du Gauteng). Puissantes économiquement et politiquement, elles intègrent de plus leurs périphéries et sont ainsi dotées d'une capacité de planification de leur croissance. En fait, ces aires métropolitaines, à l'image du pays, se trouvent écartelées entre d'une part leur mission redistributive et de rattrapage en termes de viabilisation des quartiers défavorisés et, d'autre part, la promotion de leur vocation de lieux mondialisés et émergents de production et de services. 
Illustration 6 - «Promesses » : en campagne pour les premières élections municipales postapartheid

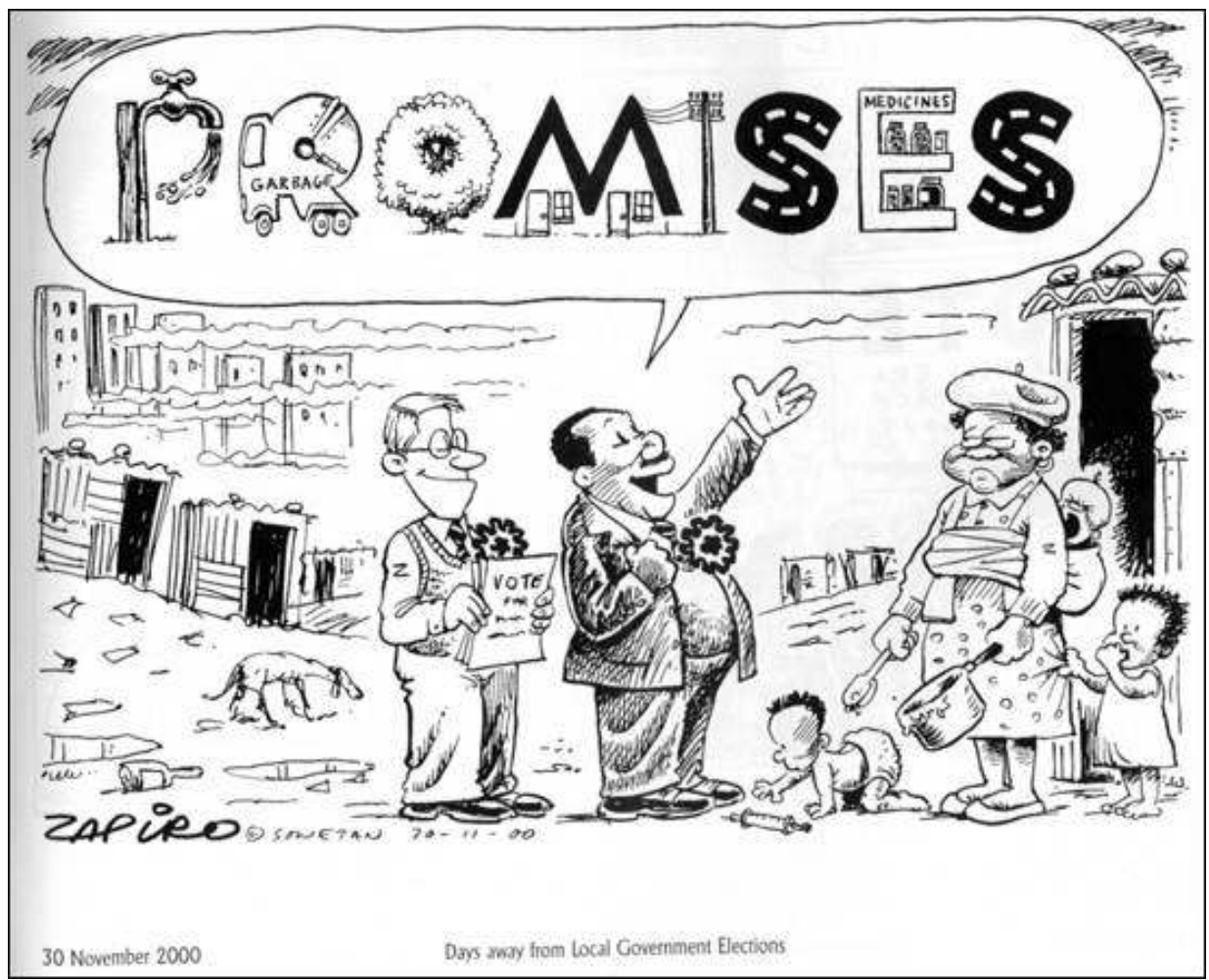

Source : dessin de Zapiro pour le quotidien national The Sowetan, 2000.

Le dispositif mis en place est donc finalement d'une extrême complexité puisqu'il faut encore ajouter les espaces stratégiques pour l'attraction des investissements directs étrangers dotés de statuts dérogatoires de concessions et de zones franches, ainsi que les éphémères municipalités transprovinciales destinées à régler des problèmes frontaliers au niveau provincial. L'entreprise de rationalisation initiale s'apparente au final à une démarche de construction pragmatique d'entités à statut différencié et éventuellement évolutif adaptées à la diversité des situations géographiques et des enjeux. On a pu qualifier cette approche et ces solutions d'innovations postmodernes en matière d'ingénierie territoriale dans la mesure où elles promeuvent une géométrie variable et s'affranchissent d'une part des principes modernes d'exhaustivité et d'emboîtement et d'autre part des principes coloniaux de hiérarchisation (incorporation sélective des lieux au domaine municipal), de spécialisation (zonage) et de cantonalisation (réserves) dans le traitement territorial de la souveraineté locale et régionale. 


\section{BIBLIOGRAPHIE}

Antheaume B. \& Giraut F., 2002. Les marges au cœur de l'innovation territoriale ? Regards croisés sur les confins administratifs (Afrique du Sud, France, Maroc, Niger, Togo ...). Historiens et géographes, HS Congrès de l'UGI de Durban : Regards sur l'Afrique, F. Bart, J. Bonvallot \& R. Pourtier (eds.), p. 39-58.

Cameron R., 2006. Local government boundary reorganization. In Democracy and Delivery, Urban Policy in South Africa, U. Pilay, R Tomlinson \& J. du Toit (eds.), Cape Town: HSRC Press, p. 76-106.

Gervais-Lambony P., 2002. Les enjeux d'une politique de redéfinition territoriale: la création de l'aire métropolitaine d'Ekurhuleni (Afrique du Sud). Autrepart, 21, p. 27-41.

Giraut F. \& Maharaj B., 2003. Contested terrains. Cities and towns in post-apartheid boundaries delimitations. Geojournal, 57(1/2), p. 15-27. http://www.springerlink.com/content/ g724732314022118/

Giraut F. \& Vacchiani-Marcuzzo C., 2009. Territories and Urbanisation in South Africa, Atlas and geo-historical information system (DYSTURB). Paris, IRD Editions (Coll. Atlas Numérique). http:// www.cartographie.ird.fr/dysturb.html

Giraut F., Guyot S. \& Houssay-Holszchuch M., 2008. Enjeux de mots : les changements toponymiques sud-africains. L'Espace géographique, 2, p. 131-150. http://www.cairn.info/revueespace-geographique-2008-2-p-131.htm

Meligrana J. (ed.), 2004. Redrawing Local Government Boundaries. An International Study of Politics, Procedures, and Decisions. Vancouver: University of British Columbia Press.

Ramutsindela M.R., 2001. Unfrozen Ground: South Africa's Contested Spaces. Aldershot, Ashgate.

Robinson J., 2008. Developing ordinary cities: visioning processes in Durban and Johannesburg. Environment and Planning, A 40, p. 74-87.

Robinson J., 2006. Ordinary cities: between modernity and development. London: Routledge.

Sutcliffe M., 1996. The Fragmented City: Durban, South Africa. In Cities of the Future: Managing Social Transformations, International Social Science Journal (Unesco), p. 67-72.

Sutcliffe M. (Chairperson of Demarcation Board), 2003. Creating cities of Hope. In Recompositions territoriales, confronter et innover. Actes des rencontres scientifiques franco-sud africaines de l'innovation territoriale, Antheaume B., Giraut F. \& Maharaj B. (dirs) http://www.pacte.cnrs.fr/ spip.php?rubrique173

\section{AUTEUR}

\section{FRÉDÉRIC GIRAUT}

Université de Genève, Frederic.Giraut@unige.ch 